\title{
A NOVEL APPLICATION OF RELAY FEEDBACK FOR PID AUTO-TUNING
}

\author{
Ioan Nascu ${ }^{(1)}$, Robin De Keyser ${ }^{(2)}$ \\ (1) Technical University of Cluj Napoca, Department of Automation \\ Baritiu 26, Cluj Napoca, 3400, Romania \\ E-mail: Ioan.Nascu@aut.utcluj.ro \\ ${ }^{(2)}$ Ghent University, EeSA-department of Electrical Energy, Systems \& Automation \\ Technologiepark 913, 9052 Gent, Belgium \\ E-mail: $\underline{\text { rdk@autoctrl.rug.ac.be }}$
}

\begin{abstract}
This paper presents a novel application of the widely used relay-feedback PIDautotuner for stable SISO processes. The proposed method consists of two steps: process identification and controller design. First, a non-iterative procedure is suggested for identification of two points on the process Nyquist curve. A second-order-plus-dead-time model is obtained and then used for PID controller design based on the internal model principle (IMC). For the identification of the two points on the Nyquist curve a pure relay in the feedback loop (as used in standard auto-tuning) and a relay which operates on the integral of the error are used. Copyright (C) 2003 IFAC
\end{abstract}

Keywords: auto-tuning, PID controller, relay experiment, Nyquist plot .

\section{INTRODUCTION}

Despite the development of more advanced control strategies, the majority of controllers used in industrial instrumentation are still of the PID type. Their popularity is easy to understand: they have a simple structure, their principle is well understood by instrumentation engineers and their control capabilities have proven to be adequate for most control loops. Moreover, due to process uncertainties, a more sophisticated control scheme is not necessarily more efficient in real-life applications than a well-tuned PID controller. However, it is common that PID controllers are often poorly tuned in practice because the choice of controller parameters requires professional knowledge by the user. To simplify this task and to reduce the time required for it, PID controllers can incorporate autotuning capabilities, i.e. they are equipped with a mechanism capable of computing the 'correct' parameters automatically when the regulator is connected to the field (Aström, et al., 1984; Aström, et al., 1992; Aström, et al., 1995; Leva, et al., 2002). Auto-tuning is a very desirable feature and almost every industrial PID controller provides it nowadays. These features provide easy-to-use controller tuning and have proven to be well accepted among process engineers.

For the automatic tuning of the PID controllers, several methods have been proposed. Some of these methods are based on identification of one point of the process frequency response, while the others are based on the knowledge of some characteristic parameters of the open-loop process step response. The identification of a point of the process frequency response can be performed either using a proportional regulator, which brings the closed-loop system to the stability boundary, or by a relay forcing the process output to oscillate. Åström and Hägglund (Aström, et al., 1984) report an important and interesting approach. Their method is based on the Ziegler-Nichols frequency domain design formula. A relay connected in a feedback loop with the process is used in order to determine the critical point.

This paper describes the development of an autotuning method based on the identification of two 
points on the process Nyquist curve: the intersection with the real negative axis and that with the imaginary negative axis. The use of two relay feedback experiments provides information to determine a four parameters second-order-plus-deadtime (SOPDT) model.

\section{THE EXPERIMENTAL SET-UP}

A standard PID control system with single input single output, as shown in figure 1 , is considered.

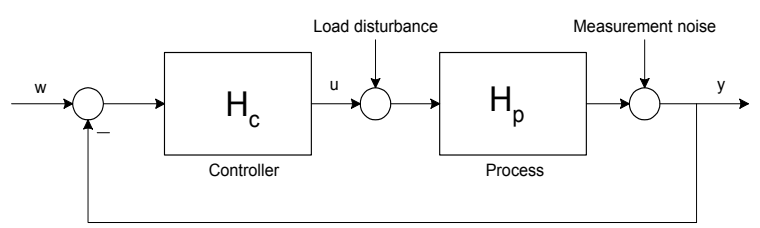

Figure 1. Control system structure

The process $H_{p}$ is assumed to be linear, stable and proper. The PID controller has a non-interacting structure cascaded with a first order filter:

$$
H_{c}(s)=K_{c}\left(1+\frac{1}{s T_{i}}+s T_{d}\right) \frac{1}{T_{f} s+1}
$$

For the purpose of PID-controller design, higher order models are of limited utility even if the process dynamics are theoretically of high order. From the control point of view, complex process models lead to complex controllers. Several papers on PID control are based on the idea of using second order with time delay models. In this paper it is assumed that the process dynamics can be described with reasonable accuracy by a second-order-plus-deadtime (SOPDT) model as:

$$
H_{p}(s)=\frac{k_{p} e^{-\theta s}}{\tau^{2} s^{2}+2 \zeta \tau s+1}, \theta \geq 0 \text { and } \tau, \zeta>0
$$

In order to tune the PID controller we will approximate $\mathrm{e}^{-\theta \mathrm{s}}$ by $(1-\theta \mathrm{s})$ and using the well-known IMC-PID design method we then obtain:

$$
\begin{aligned}
& K_{c}=\frac{2 \zeta \tau}{k_{p}\left(2 T_{c}+\theta\right)} \\
& T_{i}=2 \zeta \tau, T_{d}=\frac{\tau^{2}}{2 \zeta \tau}, T_{f}=\frac{T_{c}{ }^{2}}{2 T_{c}+\theta}
\end{aligned}
$$

thus resulting in the closed-loop transfer function:

$$
H_{0}(s)=\frac{H_{p}(s) \cdot H_{c}(s)}{1+H_{p}(s) \cdot H_{c}(s)}=\frac{e^{-\theta s}}{\left(T_{c} s+1\right)^{2}}
$$

The parameter $T_{\mathrm{c}}$ is the closed-loop time constant and is a design parameter which can be selected by the user in order to tune the controller 'aggresiveness'.
The parameters of the process transfer function (2) are assumed to be unknown and - in order to identify them - 2 points of the frequency response have to be estimated using two relay feedback experiments: the first experiment uses a relay with hysteresis or an integrating relay (= a relay without hysteresis which has as input the integral of the error) and the second experiment uses a pure relay (without hysteresis). After performing these two experiments the values of the process frequency response at two different frequencies $\omega_{1}$ and $\omega_{2}$ are obtained:

$$
H_{p}\left(j \omega_{1}\right)=a_{1}+j b_{1} \text { and } H_{p}\left(j \omega_{2}\right)=a_{2}+j b_{2}
$$

The procedure how to perform these experiments and how to obtain the values for $\left(a_{i}, b_{i} ; i=1,2\right)$ is further explained in section 3 .

\section{THE TUNING METHOD}

Note from (2) that $H_{p}(j \omega)$ can be written as follows:

$$
H_{p}(j \omega)=H_{p}^{\prime}(j \omega) H_{p}^{\prime \prime}(j \omega)
$$

with

$$
H_{p}^{\prime}(j \omega)=\frac{k_{p}}{1-\tau^{2} \omega^{2}+2 \zeta \tau j \omega}, \quad H_{p}^{\prime \prime}(j \omega)=(1-\theta j \omega)
$$

If we define - for a certain point on the Nyquist plot the magnitude, the phase angle and the real and imaginary components of $H_{p}(j \omega), H_{p}^{\prime}(j \omega)$ and $H_{p}^{\prime \prime}(j \omega)$ to be $\mathrm{M}, \alpha, \mathrm{a}, \mathrm{b}$, respectively $\mathrm{M}^{\prime}, \alpha^{\prime}, \mathrm{a}^{\prime}, \mathrm{b}^{\prime}$ and M", $\alpha "$, a", b", then (Ogata, 1995):

$$
\begin{aligned}
& \begin{array}{l}
M^{2}=a^{2}+b^{2}=M^{\prime 2} M^{\prime \prime 2}=\left(a^{\prime 2}+b^{\prime 2}\right)\left(a^{\prime \prime 2}+b^{\prime \prime 2}\right) \\
\alpha=\alpha^{\prime}+\alpha^{\prime \prime} \\
\operatorname{tg}\left(\alpha^{\prime}\right)=\frac{b^{\prime}}{a^{\prime}}=\operatorname{tg}\left(\alpha-\alpha^{\prime \prime}\right)=\frac{\operatorname{tg}(\alpha)-\operatorname{tg}\left(\alpha^{\prime \prime}\right)}{1+\operatorname{tg}(\alpha) \operatorname{tg}\left(\alpha^{\prime \prime}\right)}= \\
=\frac{\frac{b}{a}-\frac{b^{\prime \prime}}{a^{\prime \prime}}}{1+\frac{b}{a} \frac{b^{\prime \prime}}{a^{\prime \prime}}}=\frac{a^{\prime \prime} b-a b^{\prime \prime}}{a a^{\prime \prime}+b b^{\prime \prime}} \\
b^{\prime}=a^{\prime} \frac{a^{\prime \prime} b-a b^{\prime \prime}}{a a^{\prime \prime}+b b^{\prime \prime}} \quad a^{\prime}=b^{\prime} \frac{a a^{\prime \prime}+b b^{\prime \prime}}{a^{\prime \prime} b-a b^{\prime \prime}}
\end{array}
\end{aligned}
$$

From (9) - and afterwards (7) - it can be written:

$$
\begin{aligned}
& \left(a^{\prime 2}+b^{\prime 2}\right)=a^{\prime 2}\left[1+\left(\frac{a^{\prime \prime} b-a b^{\prime \prime}}{a a^{\prime \prime}+b b^{\prime \prime}}\right)^{2}\right]= \\
& =a^{\prime 2} \frac{\left(a^{\prime \prime 2}+b^{\prime \prime 2}\right)\left(a^{2}+b^{2}\right)}{\left(a a^{\prime \prime}+b b^{\prime \prime}\right)^{2}}=a^{\prime 2} \frac{\left(a^{\prime \prime 2}+b^{\prime \prime 2}\right)^{2}\left(a^{\prime 2}+b^{\prime 2}\right)}{\left(a a^{\prime \prime}+b b^{\prime \prime}\right)^{2}}
\end{aligned}
$$

thus leading to: 


$$
a a^{\prime \prime}+b b^{\prime \prime}=a^{\prime}\left(a^{\prime \prime 2}+b^{\prime \prime 2}\right)=a^{\prime} M^{\prime \prime 2}
$$

and using (9) again also to:

$$
a^{\prime \prime} b-a b^{\prime \prime}=b^{\prime} M^{\prime \prime 2}
$$

Thus for the two points obtained from the relay feedback experiments we have:

$$
\begin{aligned}
& a_{1} a_{1}^{\prime \prime}+b_{1} b_{1}^{\prime \prime}=a_{1}^{\prime} M_{1}^{\prime \prime 2} \\
& a_{1}^{\prime \prime} b_{1}-a_{1} b_{1}^{\prime \prime}=b_{1}^{\prime} M_{1}^{\prime \prime 2} \\
& a_{2} a_{2}^{\prime \prime}+b_{2} b_{2}^{\prime \prime}=a_{2}^{\prime} M_{2}^{\prime \prime 2} \\
& a_{2}^{\prime \prime} b_{2}-a_{2} b_{2}^{\prime \prime}=b_{2}^{\prime} M_{2}^{\prime \prime 2}
\end{aligned}
$$

The parameters $a_{i}$ and $b_{i}(\mathrm{i}=1,2)$ can be obtained using the two relay feedback experiments. In order to identify a point on the process Nyquist curve, a relay connected in a feedback loop with the process is used, forcing the process output to oscillate. Therefore assume that $d_{i}$ is the relay amplitude and $\varepsilon_{i}$ is the relay hysteresis width. For the given values $d_{i}$ and $\varepsilon_{i}$ we will obtain oscillation with amplitude $h_{i}$ and period $T_{i}$ in the process output.

In the first experiment there are two possibilities: using a relay with hysteresis, a point in the third quadrant on the process Nyquist curve, $P_{h}\left(a_{1}, j b_{1}\right)$, is identified (Aström, et al., 1995):

$$
\begin{array}{ll}
\omega_{1}=\frac{2 \pi}{T_{1}}, & a_{1}=\frac{-\pi \sqrt{h_{1}^{2}-\varepsilon_{1}^{2}}}{4 d_{1}}, \\
b_{1}=-\frac{\pi \varepsilon_{1}}{4 d_{1}}, & M_{1}{ }^{2}=a_{1}^{2}+b_{1}^{2}
\end{array}
$$

or, using an integrating relay $\left(\varepsilon_{1}=0\right)$ the point given by the intersection of the process Nyquist curve and the negative imaginary axis, $P_{i}\left(a_{l}, j b_{l}\right)$, is identified:

$$
\omega_{1}=\frac{2 \pi}{T_{1}}, \quad a_{1}=0, \quad b_{1}=-\frac{\pi h_{1}}{4 d_{1}}, \quad M_{1}^{2}=b_{1}^{2}
$$

In the second experiment, using a pure relay $\left(\varepsilon_{2}=0\right)$, the point given by the intersection of the process Nyquist curve and the negative real axis, $P_{p}\left(a_{2}, j b_{2}\right)$, is identified:

$$
\omega_{2}=\frac{2 \pi}{T_{2}}, \quad a_{2}=\frac{-\pi h_{2}}{4 d_{2}}, \quad b_{2}=0, \quad M_{2}^{2}=a_{2}^{2}
$$

The real and imaginary components of $H_{p}^{\prime}\left(j \omega_{i}\right)$ and $H_{p}^{\prime \prime}\left(j \omega_{i}\right)$ can be written as:

$$
\begin{aligned}
& a_{i}^{\prime}=\operatorname{Re}\left(H_{p}^{\prime}\left(j \omega_{i}\right)\right)=\frac{k_{p}\left(1-\tau^{2} \omega_{i}^{2}\right)}{\left(1-\tau^{2} \omega_{i}^{2}\right)^{2}+\left(2 \zeta \tau \omega_{i}\right)^{2}}=\frac{\left(1-\tau^{2} \omega_{i}^{2}\right) M_{i}^{\prime 2}}{k_{p}} \\
& b_{i}^{\prime}=\operatorname{Im}\left(H_{p}^{\prime}\left(j \omega_{i}\right)\right)=\frac{k_{p}\left(-2 \zeta \tau \omega_{i}\right)}{\left(1-\tau^{2} \omega_{i}^{2}\right)^{2}+\left(2 \zeta \tau \omega_{i}\right)^{2}}=\frac{-2 \zeta \tau \omega_{i} M_{i}^{\prime 2}}{k_{p}} \\
& a_{i}^{\prime \prime}=\operatorname{Re}\left(H_{p}^{\prime \prime}\left(j \omega_{i}\right)\right)=1 \quad b_{i}^{\prime \prime}=\operatorname{Im}\left(H_{p}^{\prime \prime}\left(j \omega_{i}\right)\right)=-\theta \omega_{i}
\end{aligned}
$$

Inserting in (10) the relations (13) for $a_{i}^{\prime}, b_{i}^{\prime}, a_{i}^{\prime \prime}, b_{i}^{\prime \prime} ; i=1,2$ gives 4 equations with 4 unknown parameters: $\mathrm{k}_{\mathrm{p}}, \theta, \tau^{2}$ and $2 \zeta \tau$ :

$$
\begin{gathered}
a_{1}-b_{1} \theta \omega_{1}=\frac{\left(1-\tau^{2} \omega_{1}^{2}\right) M_{1}^{2}}{k_{p}} \\
b_{1}+a_{1} \theta \omega_{1}=\frac{-2 \zeta \tau \omega_{1} M_{1}^{2}}{k_{p}} \\
a_{2}-b_{2} \theta \omega_{2}=\frac{\left(1-\tau^{2} \omega_{2}^{2}\right) M_{2}^{2}}{k_{p}} \\
b_{2}+a_{2} \theta \omega_{2}=\frac{-2 \zeta \tau \omega_{2} M_{2}^{2}}{k_{p}}
\end{gathered}
$$

Using in the first experiment a relay with hysteresis and in the second experiment a pure relay (the 'HR' case) then the first identified point on the process Nyquist curve is in the third quadrant and the second one is the intersection with the negative real axis (thus $b_{2}=0$ ). From (14.b) and (14.d) results:

$$
\theta=\frac{a_{2} b_{1}}{\omega_{1}\left(M_{1}^{2}-a_{2} a_{1}\right)}
$$

From (14.c) and (14.a) results:

$$
k_{p}=\frac{M_{1}{ }^{2} M_{2}{ }^{2}\left(\omega_{2}{ }^{2}-\omega_{1}{ }^{2}\right)}{\omega_{2}{ }^{2} M_{2}{ }^{2}\left(a_{1}-b_{1} \theta \omega_{1}\right)-a_{2} \omega_{1}{ }^{2} M_{1}{ }^{2}}
$$

Now, (14.a) and (14.b) give:

$$
\begin{gathered}
\tau^{2}=\frac{1}{\omega_{1}{ }^{2}}+\frac{k_{p}\left(b_{1} \theta \omega_{1}-a_{1}\right)}{\omega_{1}{ }^{2} M_{1}^{2}} \\
2 \zeta \tau=-\frac{k_{p}\left(b_{1}+a_{1} \theta \omega_{1}\right)}{\omega_{1} M_{1}^{2}}
\end{gathered}
$$

These values can then be used in (3) to calculate the PID-parameters.

Using in the first experiment an integrating relay and in the second experiment a pure relay (the 'IR' case) then the first identified point on the process Nyquist curve is the intersection with the negative imaginary axis (thus $\mathrm{a}_{1}=0$ ) and the second one is the intersection with the negative real axis (thus $b_{2}=0$ ). In this case we obtain the following parameters which can then be used in (3) to tune the PID: 


$$
\begin{aligned}
& \theta=\frac{a_{2}}{\omega_{1} b_{1}} \\
& k_{p}=\frac{a_{2} b_{1}^{2}\left(\omega_{1}^{2}-\omega_{2}^{2}\right)}{b_{1} \omega_{1}\left(a_{2} \theta \omega_{2}^{2}+b_{1} \omega_{1}\right)} \\
& \tau^{2}=\frac{1}{\omega_{1}^{2}}\left(1+\frac{k_{p} a_{2}}{b_{1}^{2}}\right) \\
& 2 \zeta \tau=-\frac{k_{p}}{\omega_{1} b_{1}}
\end{aligned}
$$

On the other hand, to perform relay feedback experiments, the process is first brought to steadystate conditions in manual control or with any preliminary tuned PI controller. Measuring steadystate values $\mathrm{u}_{0}$ and $\mathrm{y}_{0}$ of the controller and process output, giving then a small perturbation to the control variable and measuring its effect on the process output, the process gain $\mathrm{k}_{\mathrm{p}}$ can be easily determined instead of using (15.b) or (16.b). For processes with small nonlinearities better results are then obtained. For processes of first or second order without time delay, the value of $\theta$ in the model (2) will be zero. Strictly applying theory, these processes cannot be forced to oscillate by a pure relay. A relay without hysteresis can be used only if the process Nyquist curve crosses the negative real axis. In fact, considering that in any digital controller implementation the sampling process itself introduces a phase lag and that in real situations the process output is filtered, it can be assumed that all the processes in practical cases will oscillate when a relay controller is connected. Unfortunately, in absence of hysteresis, the relay experiment gives small values for the amplitude of oscillation, difficult to measure in the presence of measurement noise. Thus, in case of small amplitude oscillations, in the pure relay experiment $a_{2}$ and $\theta$ are fixed to zero, $k_{p}$ is computed from steady-state conditions and $\tau^{2}$ and $2 \zeta \tau$ are computed using measurements only from the first experiment $\left(\mathrm{a}_{1}, \mathrm{~b}_{1}, \omega_{1}\right)$.

If there are no others restrictions, the amplitude of the relay will be set to $0.1 \mathrm{u}_{0}$ and the hysteresis to $0.01 y_{0}$. Better results can be obtained using IR method.

\section{EXAMPLES}

First the proposed identification method has been tested. An example of the identification with both methods (HR and IR) is shown in Figure 2. The real process transfer function is given by:

$$
H_{p}(s)=\frac{2 e^{-50 s}}{(80 s+1)^{3}}
$$

We identified a second-order-plus-dead-time (SOPDT) model (equation 2). The estimated parameter values are presented in table 1 . In this example better results are obtained using an integrating relay (IR case) instead of a relay with hysteresis (HR case). Also, the computations are simpler in the IR case. In both cases better results are obtained determining $\mathrm{k}_{\mathrm{p}}$ from steady-state conditions instead of using (15.b) or (16.b). In the IR case (Fig. 2.a) the real process Nyquist plot (solid line) is not too separate from the Nyquist plot of the identified model using steady-state conditions for $k_{\mathrm{p}}$ (dashdot line)

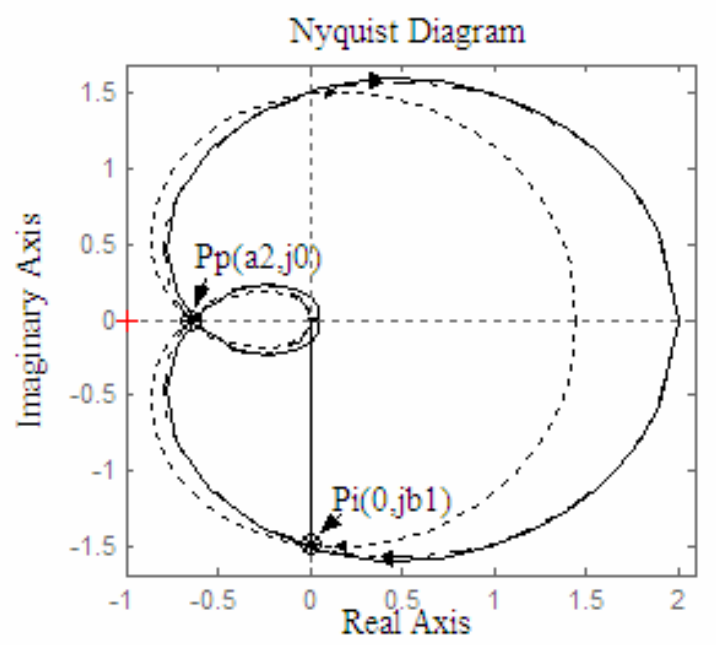

a.)

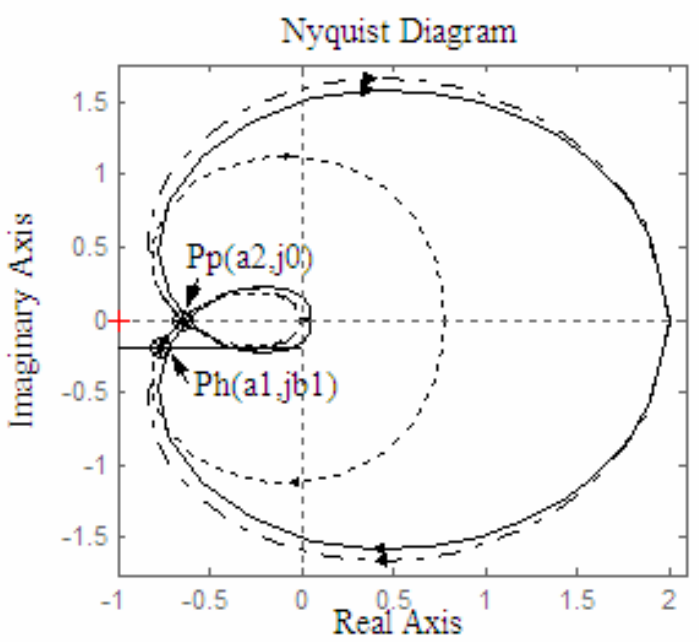

b.)

Figure 2. Nyquist plot of the process given by the transfer function (17) (solid line) and of the process model (2) identified using the two relay experiments IR/HR, with $k_{\mathrm{p}}$ computed from

\begin{tabular}{|c|c|c|c|c|c|}
\hline $\mathrm{k}_{\mathrm{p}}$ determination & Method & $\mathrm{k}_{\mathrm{p}}$ & $\theta$ & $2 \zeta \tau$ & $\tau^{2}$ \\
\hline \multirow{2}{*}{$\begin{array}{l}\text { steady-state } \\
\text { conditions }\end{array}$} & HR & 2 & 84 & 257 & 20225 \\
\hline & IR & 2 & 78 & 239 & 12878 \\
\hline \multirow{2}{*}{$\begin{array}{l}\text { relay-experiment } \\
\text { measurements }\end{array}$} & HR & 0.77 & 84 & 99 & 18012 \\
\hline & IR & 1.45 & 78 & 173 & 12217 \\
\hline
\end{tabular}
steady-state conditions (dashdot line) respectively from relay experiment (dotted line); a) IR case , b) HR case

Table 1 
The proposed auto-tuning algorithms have been tested by simulated examples. Different aspects, such as setpoint changes and load disturbances, have been analyzed. The amplitude of the setpoint and the load disturbance is 1 . The closed loop step responses using IR method are presented in the left plots and the load disturbance responses in the right plots of Figs. 3/4. The design parameter $T_{\mathrm{c}}$ can be specified by the user or can be chosen automatically as a percent of $2 \zeta \tau$ or $2 \zeta \tau+\theta$. Here $T_{\mathrm{c}}$ is fixed at $(2 \zeta \tau+\theta) / 2$ for the upper plots and respectively $(2 \zeta \tau+\theta) / 10$ for lower plots in Figs. 3/4.
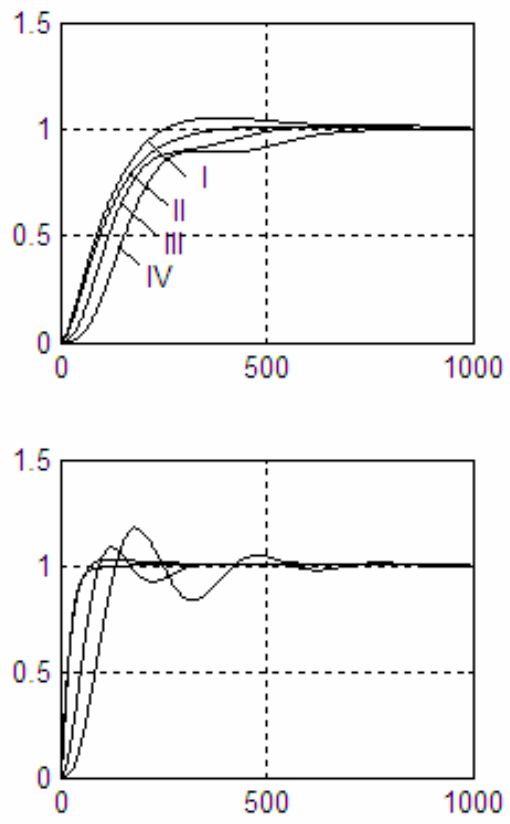

Figure 3. Responses for processes without delay
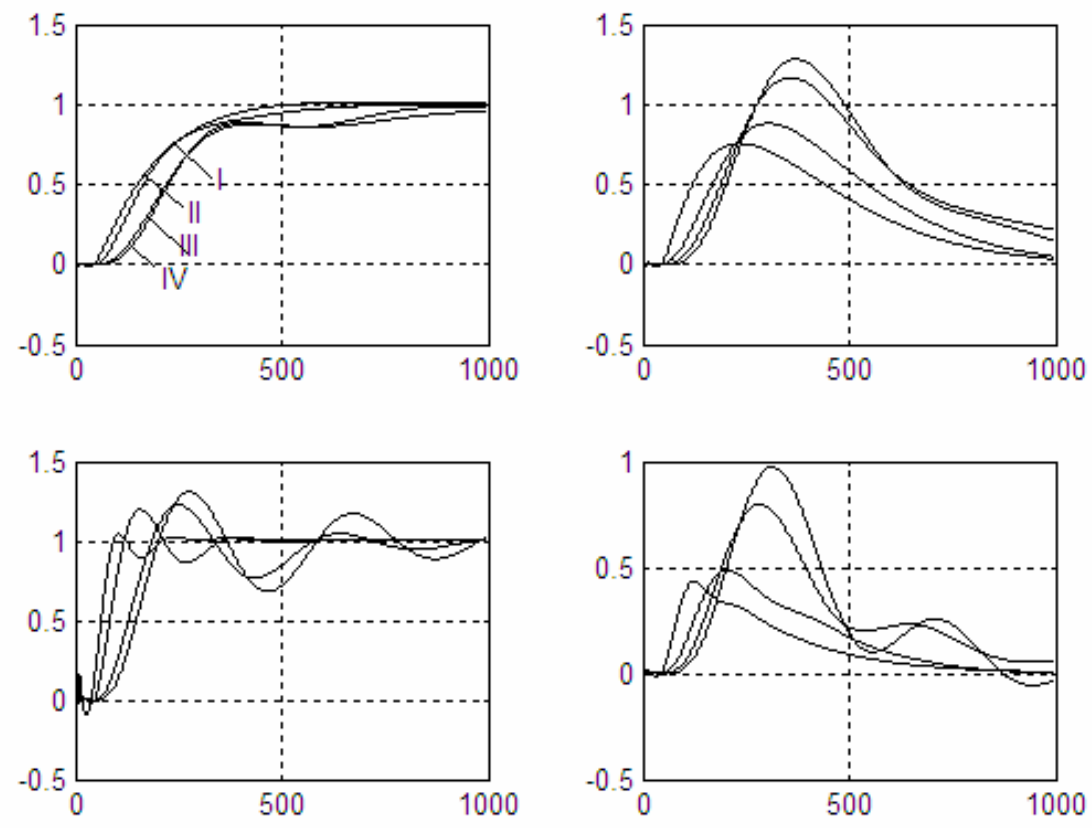

In Fig. 3 the results are presented for processes of order 1 to 4 with no delay:

$$
H_{p}(s)=\frac{2}{\left(\frac{240}{i} s+1\right)^{i}}, \quad i=1, \ldots 4
$$

In Fig. 4 the results are presented for the same processes with time delay $50 \mathrm{~s}$.
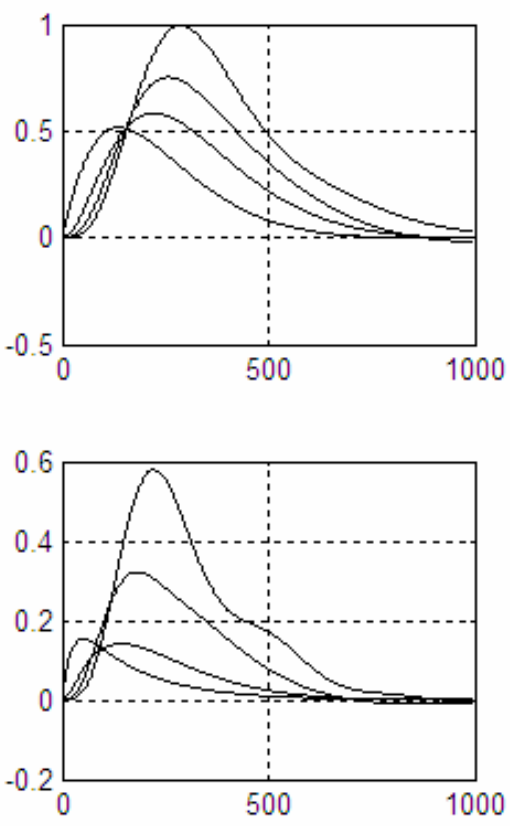

Figure 4. Responses for processes with delay 
In figure 5 and 6 the performances of the IR method are compared with two other methods: ZieglerNichols method $(\mathrm{ZN})$ and internal model control (IMC). Notice that here IMC can be considered as a kind of reference but this is not an auto-tuning method and it requires the full process model. In figure 5 a second order process given by (18) is considered. The same process with time delay $50 \mathrm{~s}$ is considered in figure $6 . T_{\mathrm{c}}$ is fixed at $(2 \zeta \tau+\theta) / 10$. The closed loop step responses are presented in the upper plots and the load disturbance responses in the lower plots.
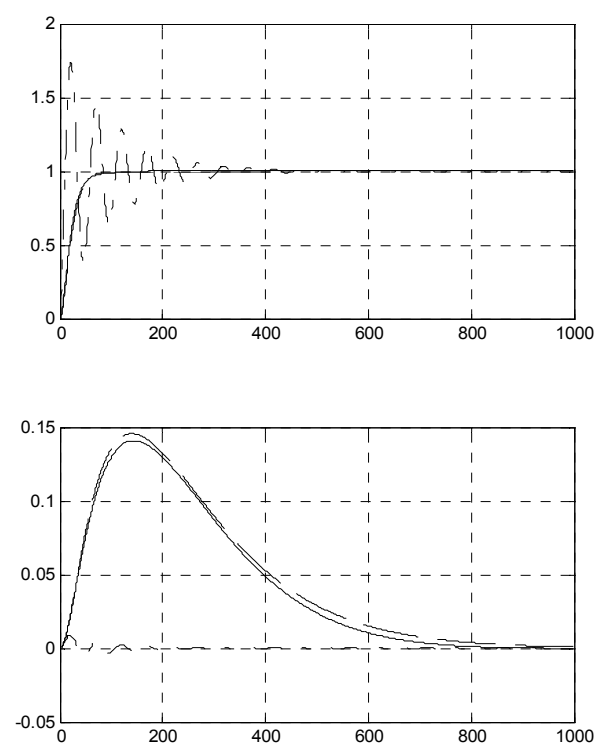

Figure 5. Comparison of the IR(solid line), IMC(dashed) and ZN (dashdot) methods, step response and load disturbance response. Second order process without delay.
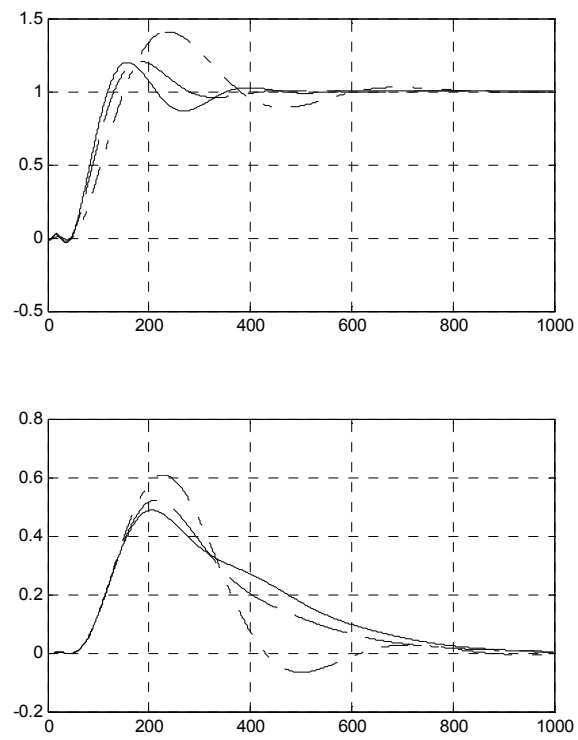

Figure 6. Comparison of the IR(solid line), IMC(dashed) and ZN (dashdot) methods, step response and load disturbance response. Second order process with delay.

\section{CONCLUSION}

Some relay-based algorithms for auto-tuning of PID controllers have been presented, assuming a simple process model structure and achieving the regulator tuning by identifying two points on the process frequency response and using the IMC-PID design method. This auto-tuning method yields good PID parameters for a specific class of processes (stable processes with self-regulation); it is not a general methodology for arbitrary process models.

Concerning the numerical complexity, the proposed method involves more complicated calculations than Ziegler-Nichols/Åström-Hägglund; however the required experiments are non-iterative and easy to be performed. Experiments and simulation studies have indicated that the presented auto-tuner performs well and can be easily used even by people who are not specialist in automatic control.

\section{REFERENCES}

Aström, K.J. and T. Hägglund (1984). Automatic tuning of simple regulators with specifications on phase and amplitude margins, Automatica, Vol.20, pp.645.

Aström, K.J., C.C. Hang, P. Persson and W.K. Ho (1992). Towards intelligent PID control. Automatica, Vol.28, pp.1,.

Aström, K.J. and T. Hägglund (1995). PID Controllers: Theory, Design and Tuning. Instrument Society of America, Research Triangle Park, NC, USA, 1995.

Leva, A., C. Cox and A. Ruano (2002). Hands-on Pid autotuning: a guide to better utilisation. IFAC Professional Brief.

Ogata,K. (1990). Modern Control Engineering. Prentice-Hall.

Ho,W.K., C.C. Hang and L.S. Cao (1995). Tuning of PID controllers based on gain and phase margin specifications, Automatica, Vol.31, No.3, pp.497.

Poulin, E., A. Pomerleau, A. Desbiens and D. Hodouin (1996). Development and evaluation of an auto-tuning and adaptive PID controller, Automatica, Vol.32, pp.71.

Schei,T.S. (1994). Automatic tuning of PID controllers based on transfer function estimation. Automatica, Vol.30, pp.1983 